\title{
Staphylococcal Scalded Skin Syndrome in an Adult on Chemotherapy
}

\author{
Jonathan J. Lee ${ }^{a}$ Hillary C. Tsibris ${ }^{c}$ Arash Mostaghimib \\ Christine G. Lian ${ }^{a}$ \\ aProgram in Dermatopathology, Department of Pathology, and bepartment of \\ Dermatology, Brigham and Women's Hospital, and 'Harvard Combined Dermatology \\ Residency Program, Harvard Medical School, Boston, Mass., USA
}

Key Words

Adult $\cdot$ Staphylococcal scalded skin syndrome $\cdot$ Chemotherapy

\section{Abstract}

Staphylococcal scalded skin syndrome is a toxin-mediated, epidermolytic condition that uncommonly affects adults. A 51-year-old man receiving chemotherapy for leukemia presented with a large geographic erosion with superficial sloughing and multiple smaller lesions elsewhere. Biopsy revealed complete subcorneal splitting with multiple detached fragments of normal-appearing stratum corneum with fragments of attached acantholytic granular keratinocytes. Mild epidermal dysmaturation was also noted. Based on these findings, the patient was started on oral cephalexin, topical mupirocin, and topical clobetasol. His lesions improved significantly over the course of 1 week.

(C) 2014 S. Karger AG, Basel

\section{Case Report}

A 51-year-old male developed a rash 2 weeks after receiving vincristine for continuation therapy of his B-cell acute lymphoblastic leukemia. The patient reported an initially developing 'pimple' in the right axilla, which subsequently expanded into a large area of redness involving the right flank and right arm. This rash was noted to be extremely painful, and he developed similar, smaller lesions elsewhere. In addition to his chemotherapy, he was 
Fig. 1. Large, tender, erythematous plaque with superficial sloughing and erosion in the right axilla.
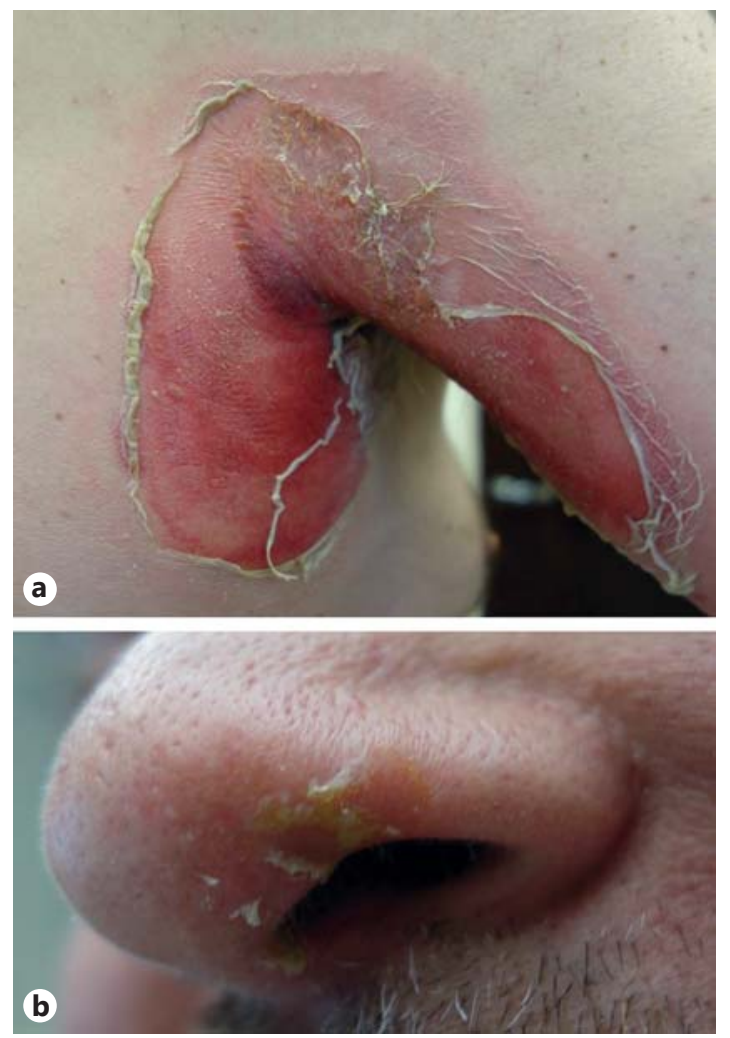

receiving 6-mercaptopurine as well as acyclovir and dapsone for prophylaxis. He denied recent fevers, chills, and/or malaise. His past medical history was significant for gout and diabetes mellitus.

Physical examination revealed a large, round, geographic erosion with superficial sloughing within the right axilla (fig. 1a). There were multiple additional tender, circular, erythematous erosions on the distal right arm and contralateral axilla, and multiple, small resolving erythematous, crusted erosions on his left nare (fig. 1b) and around his right eyelid with areas of desquamation. A sample from the axillary erosion was sent for microbiologic culture, and a biopsy of one of the circular erythematous lesions on the arm was performed.

Microbiologic culture from the axillary lesion grew 4+ Staphylococcus aureus. Histopathologic examination revealed complete subcorneal splitting of the epidermis with detachment of the stratum corneum (fig. 2a). Multiple detached fragments of normal-appearing stratum corneum with attached acantholytic granular keratinocytes were present (fig. 2b). There was a sparse perivascular lymphocytic infiltrate in the superficial dermis (fig. 3a). The epidermis showed subtle irregular orientation of the stratum basale and loss of normal, gradual keratinocyte flattening towards the surface, consistent with mild epidermal dysmaturation and suggestive of a chemotherapy effect (fig. $3 \mathrm{~b}$ ). In addition, closer examination of the superficial layer of granular and spinous keratinocytes underlying the detached fragments of stratum corneum revealed a cytomorphology similar to that of the basal keratinocytes. Gram stains were performed but did not reveal any microorganisms.

The histologic differential diagnoses included toxic epidermal necrolysis, bullous impetigo, pemphigus foliaceus, staphylococcal scalded skin syndrome (SSSS, or staphylococcal epidermolytic toxin syndrome), and toxic erythema of chemotherapy (TEC). Appropriate clinicopathological correlation is critical to render the correct diagnosis. Toxic 

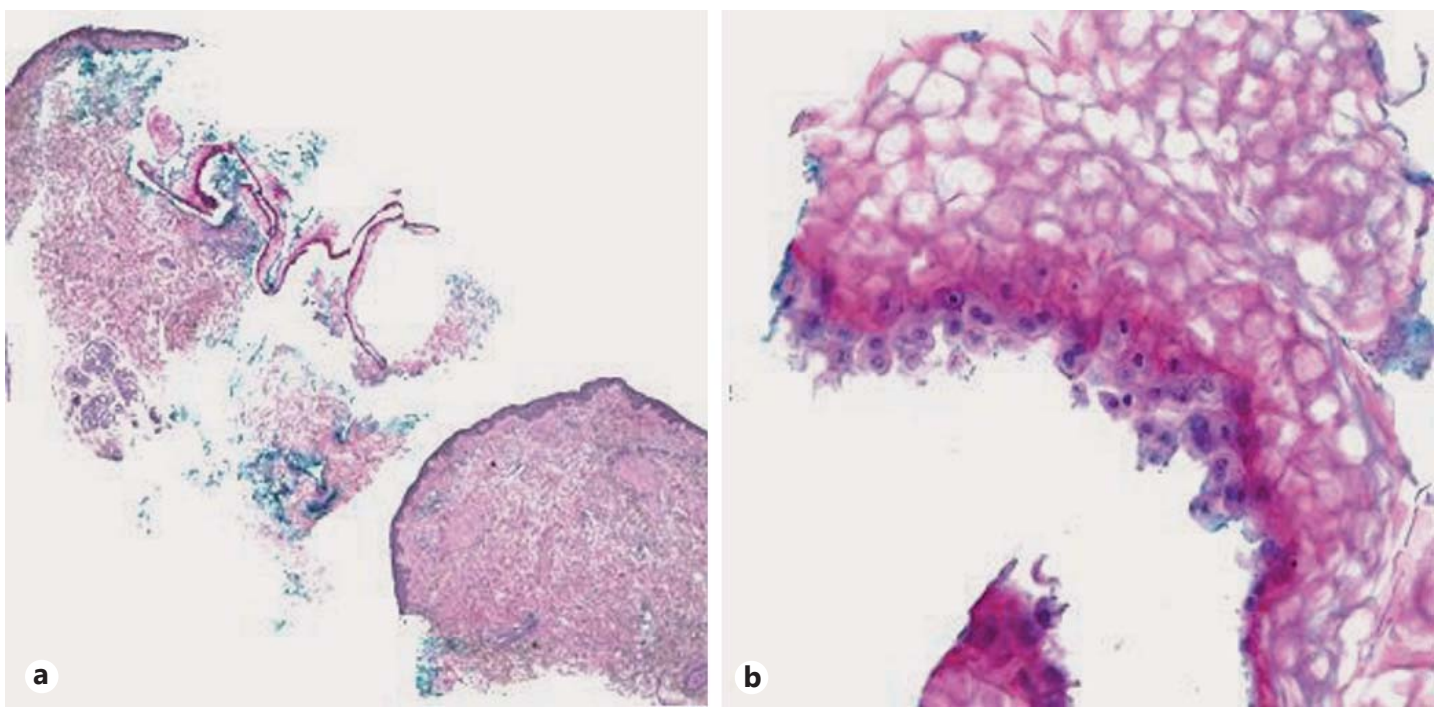

Fig. 2. Microphotographs. a Subcorneal splitting of the epidermis with multiple fragments of detached stratum corneum. HE. Scanning magnification, $\times 2$. b Strips of separated stratum corneum with attached acantholytic granular keratinocytes. HE. High-power view, $\times 100$.
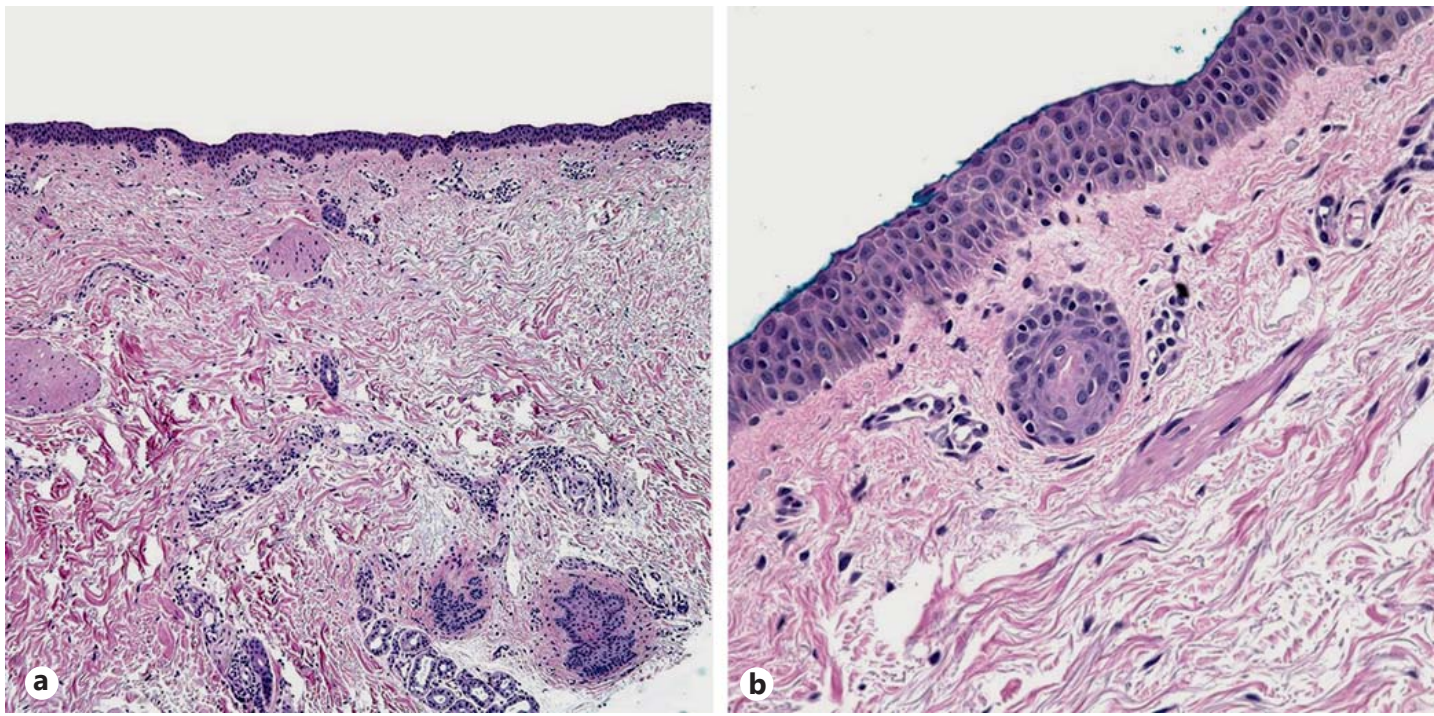

Fig. 3. Microphotographs. a Complete loss of the stratum corneum and subtle epidermal dysmaturation with mininal dermal inflammation and sparse superficial perivascular lymphocytic infiltrate. HE. Low-power view, $\times 10$. $\mathbf{b}$ Complete loss of the stratum corneum. Minimal spongiosis and epidermal dysmaturation are noted. Squamous metaplasia of the eccrine duct in the superficial dermis is present. HE. High-power view, $\times 40$.

epidermal necrolysis was less favored given the absence of mucosal involvement clinically and the lack of epidermal necrosis histologically. Secondly, bullous impetigo was thought to be less likely given the absence of organisms on Gram stain, paucity of inflammation, and absence of a neutrophilic infiltrate. Pemphigus foliaceus was also less favored given the acantholytic keratinocytes attached to the detached stratum corneum as well as the paucity of 
Fig. 4. Resolving erosion within the axilla (1 week after treatment).

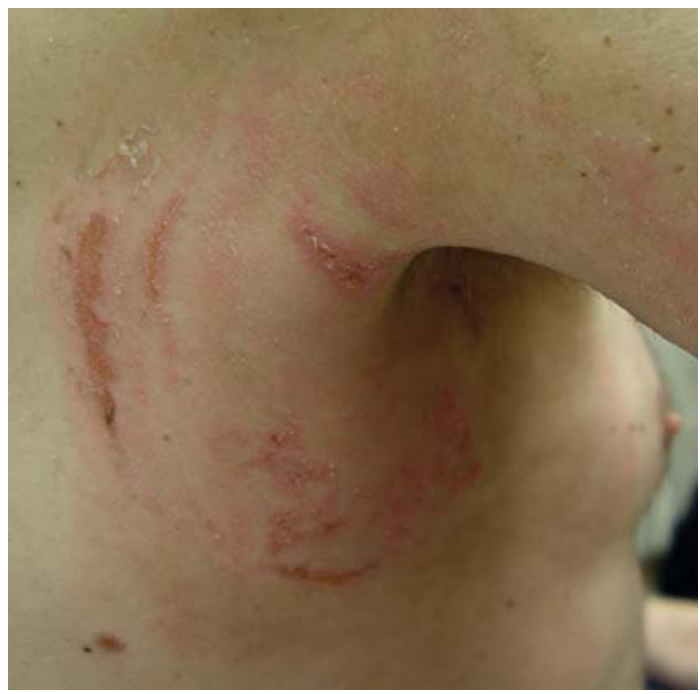

inflammation. Finally, while the mild epidermal dysmaturation present was suggestive of a chemotherapy effect, this finding alone was insufficient for a diagnosis of TEC. Characteristic diagnostic features of TEC, such as vacuolar change at the dermal-epidermal junction, eccrine ducts, and/or hair follicles as well as syringosquamous metaplasia, were not identified. Taken together, the histologic findings were most consistent with SSSS on a background of a chemotherapy effect.

Based on these findings, the patient was started on oral cephalexin as well as topical mupirocin and clobetasol. An oral pain medication was added as well. The patient's skin lesions and pain significantly improved over the course of 1 week (fig. 4).

\section{Discussion}

SSSS is a toxin-mediated, epidermolytic condition caused by the remote production and hematogenous circulation of exfoliative toxins (exfoliatoxin) that bind and cleave desmoglein 1 in the superficial epidermis. Multiple serotypes of the exfoliatoxin have been identified [1]. SSSS is a condition that predominantly affects young children and neonates (Ritter's disease), in whom the prognosis is quite favorable [2]. Nosocomial outbreaks of SSSS in neonates within a maternity unit have been described [3]. SSSS much less commonly affects adults, who typically have underlying renal dysfunction, malignancy, or immunosuppression and are thought to have a reduced ability to excrete the toxin [4]. There has been one prior report of SSSS occurring in a patient shortly after receiving chemotherapy for a peripheral neuroectodermal tumor [5]. However, cases of SSSS occurring in healthy adults have also been reported [6]. Several cases of SSSS caused by methicillin-resistant $S$. aureus in adults have been reported [7]. The prognosis of SSSS is much less favorable in adults than it is in children, and mortality rates between 40 and $50 \%$ have been reported $[2,8]$.

SSSS is a relatively uncommon condition, with epidemiologic studies estimating a yearly incidence of less than one case (0.09-0.56) per one million inhabitants per year in developed countries [2,9]. SSSS as well as localized and generalized bullous impetigo belong to the 'staphylococcal epidermolytic toxin' syndromes. While localized and generalized bullous impetigo are the result of local production of exfoliative toxins within the skin, toxin production in SSSS is classically thought to occur at a distant site. Clinically, patients with SSSS develop a 
tender, erythematous, scarlatiniform eruption followed by the development of flaccid, easily ruptured bullae involving the periorificial face, neck, axillae, trunk, and groins with sparing of the mucous membranes [10]. The bullae typically exhibit a positive Nikolsky sign, leaving behind moist, red erosions. Desquamation of the skin occurring in sheet- or ribbon-like fashion follows [11].

SSSS is characterized histologically by subcorneal splitting within the stratum granulosum [11]. The blister roof is comprised of stratum corneum and few granular keratinocytes. Rare acantholytic cells and sparse neutrophils may be present within the intact lesion. The superficial dermis typically contains a sparse mixed inflammatory infiltrate, in contrast to bullous impetigo and pemphigus foliaceus, wherein the infiltrate is typically heavier. In addition, the subcorneal blister of bullous impetigo typically contains a heavier neutrophilic infiltrate as well as multiple Gram-positive cocci [11]. The diagnosis of SSSS is typically rendered based on the clinical, histologic, and microbiologic data. Systemic and topical antibiotics targeting Gram-positive cocci in addition to appropriate wound care of erosions are the mainstay of therapy.

The histologic findings of mild epidermal dysmaturation in our patient are consistent with those seen secondary to chemotherapy. The entity 'TEC' has recently been proposed to unify a broad, heterogeneous disorder encompassing the clinical and histopathologic findings seen in chemotherapy-associated acral erythema, neutrophilic eccrine hidradenitis, and syringosquamous metaplasia [12]. Clinically, patients with TEC develop painful, pruritic erythematous or edematous patches or plaques on the hands and feet, intertriginous areas, elbows, knees, and/or ears that appear days to weeks after the administration of a number of chemotherapeutic agents [12]. These lesions may develop a dusky hue, petechiae, or bullae, and desquamate and spontaneously resolve without specific therapy. Histologically, TEC represents a 'pain-epithelial toxic disorder' characterized by vacuolar change at the dermalepidermal junction, eccrine ducts, and/or hair follicles with epidermal dysmaturation and syringosquamous metaplasia [13]. Acantholysis and follicular hyperkeratosis may also be seen. While clear vacuolar changes were rare in our patient, the findings of epidermal dysmaturation were consistent with a chemotherapy effect. Taken together, our patient represents an uncommon case of SSSS in an adult occurring on a background of chemotherapy-related changes.

\section{Disclosure Statement}

The authors have no conflicts of interest to declare.

\section{References}

1 Yamasaki O, Yamaguchi T, Sugai M, Chapuis-Cellier C, Arnaud F, Vandenesch F, et al: Clinical manifestations of staphylococcal scalded-skin syndrome depend on serotypes of exfoliative toxins. J Clin Microbiol 2005;43: 1890-1893.

2 Mockenhaupt M, Idzko M, Grosber M, Schöpf E, Norgauer J: Epidemiology of staphylococcal scalded skin syndrome in Germany. J Invest Dermatol 2005;124:700-703.

3 El Helali N, Carbonne A, Naas T, Kerneis S, Fresco O, Giovangrandi Y, et al: Nosocomial outbreak of staphylococcal scalded skin syndrome in neonates: epidemiological investigation and control. J Hosp Infect 2005;61: 130-138.

4 Cribier B, Piemont Y, Grosshans E: Staphylococcal scalded skin syndrome in adults. A clinical review illustrated with a new case. J Am Acad Dermatol 1994;30:319-324.

5 Prabhash K, Babu KG, Ravi S, Girish MH, Bapsy PP: Staphylococcal scalded skin syndrome. Lancet Infect Dis 2003;3:442. 
6 Patel GK, Varma S, Finlay AY: Staphylococcal scalded skin syndrome in healthy adults. Br J Dermatol 2000;142: 1253-1255.

7 Acland KM, Darvay A, Griffin C, Aali SA, Russell-Jones R: Staphylococcal scalded skin syndrome in an adult associated with methicillin-resistant Staphylococcus aureus. Br J Dermatol 1999;140:518-520.

8 Farrell AM, Ross JS, Umasankar S, Bunker CB: Staphylococcal scalded skin syndrome in an HIV-1 seropositive man. Br J Dermatol 1996;134:962-965.

-9 Lamand V, Dauwalder O, Tristan A, Casalegno JS, Meugnier H, Bes M, et al: Epidemiological data of staphylococcal scalded skin syndrome in France from 1997 to 2007 and microbiological characteristics of Staphylococcus aureus associated strains. Clin Microbiol Infect 2012;18:E514-E521.

10 Grayson W: Staphylococcal scalded skin syndrome; in Calonje E, Brenn T, Lazar A, McKee PH (eds): McKee's Pathology of the Skin, ed 4. Philadelphia, Elsevier Saunders, 2012, pp 791-794.

11 Weedon D: Staphylococcal 'scalded skin' syndrome; in Weedon D: Weedon's Skin Pathology, ed 3. London, Churchill Livingstone Elsevier, 2010, pp 549-550.

12 Bolognia JL, Cooper DL, Glusac EJ: Toxic erythema of chemotherapy: a useful clinical term. J Am Acad Dermatol 2008;59:524-529.

13 McCalmont TH: Tec. J Cutan Pathol 2013;40:785-787. 